Review Article

\title{
Exercise effects on physiological function during aging
}

\author{
Zsolt Radak ${ }^{\mathrm{a}, \mathrm{g}, *}$, Ferenc Torma $^{\mathrm{a}}$, Istvan Berkes ${ }^{\mathrm{a}}$, Sataro Goto ${ }^{\mathrm{b}}$, Tatsuya Mimura ${ }^{\mathrm{c}}$, Aniko Posa ${ }^{\mathrm{d}}$, \\ Laszlo Balogh $^{\mathrm{e}}$, Istvan Boldogh ${ }^{\mathrm{f}}$, Katsuhiko Suzuki ${ }^{\mathrm{g}}$, Mitsuru Higuchi ${ }^{\mathrm{g}}$, Erika Koltai ${ }^{\mathrm{a}}$
}

${ }^{a}$ Research Institute of Sport Science, University of Physical Education, Budapest, Hungary

${ }^{\mathrm{b}}$ Graduate School of Health and Sports Science, Juntendo University, Chiba, Japan, Hungary

${ }^{\mathrm{c}}$ Faculty of Sport and Health Sciences, Osaka Sangyo University, Osaka, Japan

${ }^{\mathrm{d}}$ Department of Physiology, Anatomy and Neuroscience, University of Szeged, Szeged, Hungary

${ }^{\mathrm{e}}$ Institute of Sport Science, University of Debrecen, Debrecen, Hungary

${ }^{\mathrm{f}}$ Department of Microbiology and Immunology, University of Texas Medical Branch at Galveston, Galveston, TX 77555, USA

${ }^{\mathrm{g}}$ Faculty of Sport Sciences, Waseda University, Tokorozawa, Saitama, Japan

A R T I C L E I N F O

\section{Keywords:}

Exercise

VO2max

Mitochondrial dynamics

Cellular housekeeping

\begin{abstract}
A B S T R A C T
The decrease in cognitive/motor functions and physical abilities severely affects the aging population in carrying out daily activities. These disabilities become a burden on individuals, families and society in general. It is known that aging conditions are ameliorated with regular exercise, which attenuates the age-associated decline in maximal oxygen uptake (VO2max), production of reactive oxygen species (ROS), decreases in oxidative damage to molecules, and functional impairment in various organs. While benefits of physical exercise are welldocumented, the molecular mechanisms responsible for functional improvement and increases in health span are not well understood. Recent findings imply that exercise training attenuates the age-related deterioration in the cellular housekeeping system, which includes the proteasome, Lon protease, autophagy, mitophagy, and DNA repair systems, which beneficially impacts multiple organ functions. Accumulating evidence suggests that exercise lessens the deleterious effects of aging. However, it seems unlikely that systemic effects are mediated through a specific biomarker. Rather, complex multifactorial mechanisms are involved to maintain homeostatic functions that tend to decline with age.
\end{abstract}

\section{Introduction}

Physical exercise can increase blood flow to working skeletal muscle by up to 100 - fold, and more moderately to the brain, whereas oxygen supply and circulation decrease to the liver, kidney and testes [1]. Despite the variability in oxygen, blood, and energy supply to these organs, all of them benefit from regular exercise. Indeed, regular exercise/physical activity has the powerful effect of decreasing the incidence of a wide range of lifestyle-related diseases, and the effect appears to be systemic. What are the molecular mechanism behind these systemic effects? Are they regulated by a common molecular pathway or a molecular biomarker? The answers to these very important questions are not known presently, but a reasonable suggestion would be that they result from the sum effects of many signaling pathways that are activated by exercise. An examination of the factors that influence maximal oxygen uptake $\left(\mathrm{VO}_{2} \mathrm{max}\right)$ is promising to pinpoint the key pathways or proteins involved, since the level of $\mathrm{VO}_{2} \mathrm{max}$ has become an important biomarker for quality of life that is strongly associated with a decreased incidence of lifestyle-related diseases [2-4]. It has been suggested that a high $\mathrm{VO}_{2} \max$ was important to early humans, because it probably increased the efficiency of hunting when pursuing prey [5]. Moreover, a greater aerobic capacity could have provided a significant advantage in obtaining more and higher quality food, and for escaping from enemy attack. Therefore, it is suggested that aerobic exercise, especially running, could have been important for the evolution of Homo sapiens [6,7]. Based on these observations, we suggest that not only an inherited high endurance capacity, but also the inherent ability to increase aerobic endurance capacity in response to aerobic activity, influences brain function and probably the function of other organs.

In addition, it is well established that adaptive responses to exercise could be dependent on genetic, and probably epigenetic factors [8]. The adaptive response covers the antioxidant- and oxidative damage repair systems as well as the capacity to generate reactive oxygen species (ROS) [7].

Increased levels of $\mathrm{VO}_{2}$ max not only decrease the incidence of

\footnotetext{
* Correspondence to: Institute of Sport Science, Research Institute of Sport Science, University of Physical Education, Alkotas u. 44, Budapest H-1123, Hungary.

E-mail address: radak@tf.hu (Z. Radak).
} 
mortality [4] and oxidative stress [9] but also up-regulate molecular pathways, which enhance endogenous protection against ROS [1]. On the other hand, aging is associated with decreases in $\mathrm{VO}_{2} \mathrm{max}$ and loss of organ function as well as increases in ROS production and oxidative stress [10].

In this article, we aim to critically review the scientific evidence and potential mechanisms for the systemic effects of exercise and whether ROS-related pathways are a possible explanation.

\section{Is $\mathrm{VO}_{2}$ max a marker of systemic adaptive response?}

It is clear that the level of physical fitness is an independent predictor of mortality [11] including those caused by coronary heart diseases [3,4] cancer [12], alcoholic cirrhosis [13], and diabetes [14] among others. $\mathrm{VO}_{2} \mathrm{max}$ is one of the best known and most well studied markers of physical fitness and it is well accepted that increases in $\mathrm{VO}_{2}$ max decrease the overall rate of mortality [11]. There are few studies on the relationship between $\mathrm{VO}_{2}$ max and kidney, lung and liver function, but the results suggest that low $\mathrm{VO}_{2} \mathrm{max}$ is associated with impaired function of these organs [15-17].

Moreover, it is well documented that regular exercise promotes ideal brain function [18] and mild physical activities such as walking can reduce the risk of dementia, however, the possible link between VO2max and brain function in humans has yet to be mechanically examined. On the other hand, because VO2max represents cardiovascular fitness the link between endurance and heart function is obvious. Therefore, one can hypothesize that an elevated level of $\mathrm{VO}_{2} \mathrm{max}$ is associated with the improvement or maintenance of heart, muscle, brain, lung, liver and kidney functions in middle aged and older humans.

Regular endurance exercise does increase $\mathrm{VO}_{2}$ max. However, it is known that about $15-20 \%$ of the population does not respond to exercise training with a significantly enhanced $\mathrm{VO}_{2} \max$ [19]. Thus, it is questionable whether organ function in these individuals would change.

$\mathrm{VO}_{2}$ max is dependent upon cardiac output and arterio-venous difference, and the latter is further influenced by capillarization, myoglobin levels, mitochondrial content and function. There is a close relationship between the activity of citrate synthase (CS), which is used as a marker of cellular mitochondrial content, and the levels of $\mathrm{VO}_{2} \mathrm{max}$ [20], but CS is not related to mortality, suggesting a complex adaptive regulation. In addition, the mitochondrial-specific respiration capacities during $\beta$-oxidation, including maximal oxidative phosphorylation, and electron transport system capacity, all correspondingly improve with aerobic capacity [20].

Davies at al. [21] and others [22] have shown that mitochondrial factors are more closely influence the endurance capacity than $\mathrm{VO}_{2}$ max. Moreover, when subjects with similar VO2max but different levels of physical activity were compared, it turned out that those who did regular training had a better metabolic profile, like insulin tolerance and lipid profile, and at exercise tests they over-performed those, who had similar VO2max but were untrained [23].

When the relationship between VO2max and mitochondrial haplogroups were studied, results revealed that $\mathrm{VO}_{2}$ max differences among haplogroups can account for electron transport chain function dissimilarities and production of ROS [24]. Samples were haplo-grouped by PCR amplification of short mtDNA fragments, followed by restriction enzyme analysis and confirmed by sequencing of the hypervariable region (HVR)-I and HVR-II [25]. J-haplogroup variants have higher $\mathrm{VO}_{2}$ max than individuals with no-J variants, but regular exercise training suppresses the influence of mitochondrial background. The ratio between ATP and ROS production in OXPHOS coupling efficiency seems to be the main determinant of $\mathrm{VO}_{2}$ max differences between haplogroups [24]. The H-mitochondrial haplogroup variant induces a larger amount of ROS, and this haplotype is under-represented (a small number of subjects were in this haplogroup), while J-variants are over- represented in the elderly [26]. These results suggest that mitochondria, might be one of the cellular organelles that influences the beneficial effects of the optimal $\mathrm{VO}_{2}$ max in mortality and well-being and may contribute to systemic effects of exercise at the examined population

\section{Mitochondrial dynamics in exercise and aging}

Mitochondrial content, dynamics and function, influence the generation of ROS and the overall capacity of the antioxidant system. It is known, from the pioneering work of Dr. John Holloszy, that endurance exercise increases the mass of mitochondria in the skeletal muscle [27]. Exercise training also increases mitochondrial levels in the brain, heart, liver, and kidney $[28,29]$. Moreover, exercise training increases the mtDNA content in testes, especially in aged rats [30], suggesting that exercise has a beneficial impact on male fertility, which could be influenced by mitochondrial mass and function.

The coactivator peroxisome proliferator-activated receptor- $\gamma$ coactivator $1 \alpha$ (PGC-1 $\alpha$ ), through the co-activation of nuclear respiratory factors 1 and 2 (NRF-1 and NRF-2, respectively) results in enhanced transcription of nuclear-encoded gene products that are obligatory to mitochondrial biogenesis. Besides stimulating the DNA binding of nuclear factors, PGC-1 $\alpha$ activates mitochondrial transcription factor A (TFAM), which is essential for gene expression from mtDNA [31]. During exercise, PGC- $1 \alpha$ can be activated by AMP-activated protein kinase (AMPK), silent mating type information regulation 2 homolog 1 (SIRT1), $\mathrm{Ca}^{2+}$ /calmodulin-dependent protein kinase IV, calcineurin A, and ROS $[32,33]$. But PGC- $1 \alpha$ is not just activated by ROS, it can control the level of ROS partly through the activation of nuclear factor (erythroid-derived 2)-like 2 that binds to antioxidant response element (ARE) [34]. Besides the activation of ARE, PGC-1 $\alpha$ might be important to exercise-induced increases in the expression of SOD2 [35]. In support of this hypothesis, PGC-1 $\alpha$ knock out mice exhibit a shift from oxidative type I and IIa toward type IIx and IIb muscle fibers [36], and these fibers have lower levels of antioxidant capacity than type I fibers. This shift results in decreased endurance capacity. In accordance with this down-regulation, PGC-1 $\alpha$ blunts antioxidant capacity [37]. It is also known that type I fibers have greater DNA repair enzyme activity, suggesting better housekeeping of DNA [38].

Besides autophagy and mitophagy the mitochondrial network is under continuous remodeling via mitochondrial fusion and fission, which is also influenced by aging and exercise [39]. It has been suggested that damaged mitochondria can lose their capacity to prevent exacerbation of damage through fusion when incorporating with a healthy mitochondrial network. Indeed, damaged mitochondria, having lost the mitochondrial membrane potential, are degraded, while healthy daughter mitochondria are able to proceed to fusion in order to strengthen the mitochondrial network. When young and old rats were subjected to exercise training it was observed that NRF1, TFAM, mitofusin-1 (Mfn1) and fission-1 (Fis1) levels increased with age in skeletal muscle [39]. This might be a compensatory mechanism to offset the increasing levels of dysfunctional mitochondria, as fission and fusion can dilute mitochondrial damage. Indeed high intensity interval training in addition to PGC-1 $\alpha$, significantly increased fusion and fission proteins. After seven training sessions, Fis1 was increased by more than 2 fold and dynamin-related protein-1 (DRP-1) increased by $47 \%$ [40]. Besides Mfn1, the role of Mfn2 in mitochondrial dynamics is even more significant since repression of Mfn2 leads to morphological fragmentation of the mitochondrial network, and in myotubes it causes a drop of mitochondrial membrane potential greater than $50 \%$ and glucose oxidation by at least $30 \%$ [41]. Mutation of Mfn2 is associated with myopathy [42] indicating that altered mitochondrial dynamics compromises the function of this organelle [41]. It has been shown that endurance training increased mitochondrial fusion through the activation of Mfn2 [43-45] and it appears that expansion of the volume density of mitochondria is dependent on Mfn2. Optic atrophy 1 (OPA 1) 


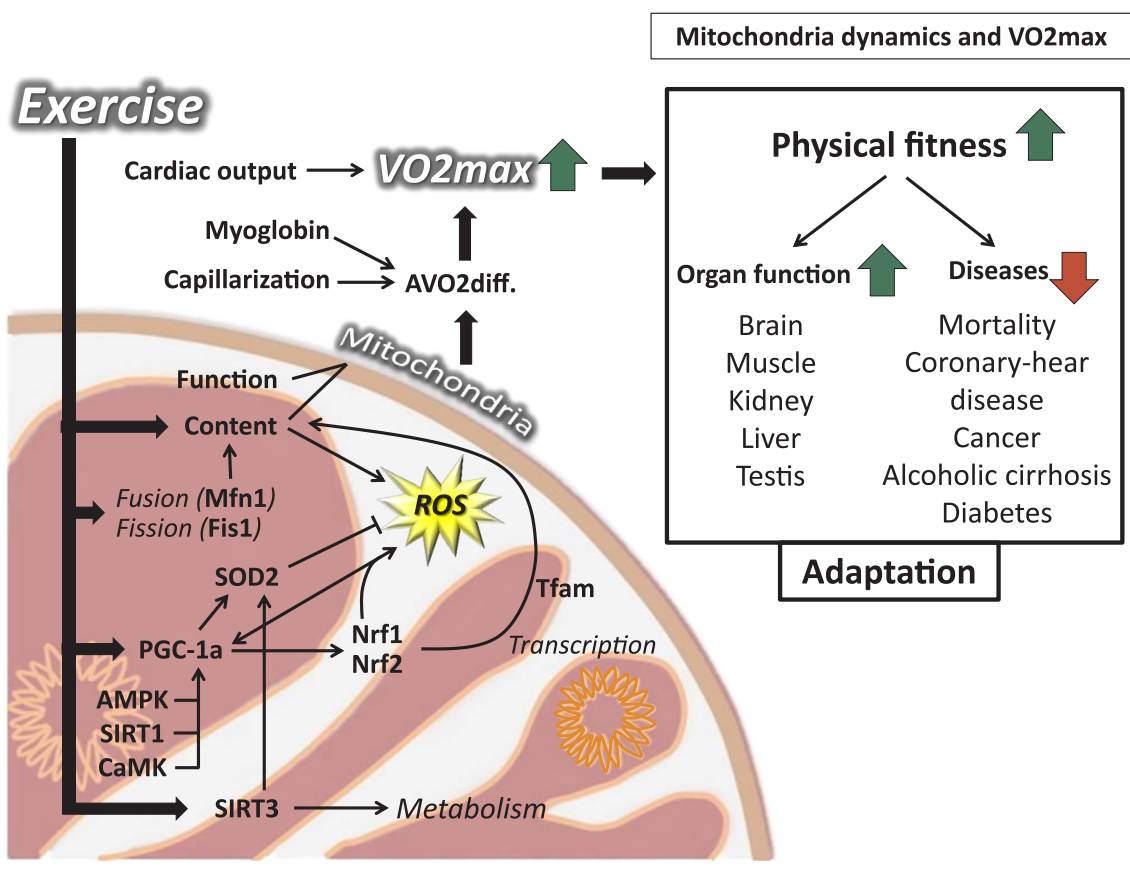

Fig. 1. The suggested relationship between VO2max, organ function and mitochondrial dynamics. Increased levels of cardiovascular fitness, VO2max is associated with decreases in mortality and improved organ function, suggesting a systemic response to regular exercise. Increased levels of fitness are often associated with mitochondrial biogenesis and well balanced mitochondrial dynamics. The adaptive response of SIRT3 to exercise training could attenuate the age-associated decline in cellular metabolism and the increase in mitochondrial ROS production. Exercise training decreases oxidative stress and attenuates age-associated deterioration of mitochondrial function.

also regulates fusion by shaping the level of inner-membrane ultrastructure and bioenergetics [46]. It has been shown that both electric stimulation and leg press training results in similar adaptation of skeletal muscle in aged subjects, which includes elevated levels of force generation, increased muscle mass, OPA1 and mitochondrial protein contents [47]. Kitaoka and co-workers reported that caloric restriction and endurance training together increased the levels of OPA1 and Mfn1 in rat skeletal muscle [48]. However, it is important to note that OPA1 can be involved in mitophagy as well, because mutation of OPA1 leads to mitophagy and excessive mitochondrial fragmentation [49].

Exercise training seems to block the age-associated increase in all of the above listed factors. Since exercise training significantly decreases the gap between young and aged muscles in the levels of various mitochondrial markers, NRF1, TFAM, Mfn1, Mfn2, OPA-1, DRP-1 and Fis1, we propose that regular exercise could help delay many of the ageassociated changes in muscle mitochondria [39]. Similar beneficial effects of regular exercise have been reported for young and old mice with ablation of PGC-1 $\alpha$, when hyper-fragmentation of mitochondrial networks was observed in aged relative to young animals, and exercise training normalized mitochondrial network structure in wild, but not in PGC-1 $\alpha$ KO animals [50] or in human subjects [51]. The beneficial effects of exercise on mitochondrial dynamics is not limited to skeletal muscle, but also includes brains [29,52,53] and fetal hearts from exercised dams [54], while other organs have not been studied.

Endurance exercise- induced increases in $\mathrm{VO}_{2} \mathrm{max}$ are associated with enhanced mitochondrial biogenesis, the antioxidant system, organ function and decreased mortality. $\mathrm{VO}_{2}$ max is referred to as cardiovascular fitness, but it is also dependent on mitochondrial mass and function. However, the work of Boushel et al., 2011 [55], revealed that mitochondrial respiratory capacity at state 3 , when half or more of the muscle mass is working, exceeds the rate of oxygen delivery capacity of the circulatory system. This result suggests that not muscle but the capacity of oxygen delivery by the cardiovascular system is the limiting factor of performance. Indeed, the ablation of single regulatory transcription factors, which influence mitochondrial biogenesis, often leads to a decrease in the basal mitochondrial content of muscle. This decrease can be overcome with exercise training, at least in part, suggesting that exercise activates a multitude of pathways, which respond to restore mitochondrial health [56].

\section{SIRT3, a mitochondrial protein that mediates exercise- associated adaptation}

Sirtuins (SIRT1-7) play an important role in aging and in the adaptation to caloric restriction and exercise by controlling vital cellular signaling pathways $[57,58]$. Recently, SIRT-3 has emerged as an important protein in mitochondrial function, as it is localized in mitochondria and, via deacetylation of regulatory proteins, controls fatty acid oxidation, oxidative phosphorylation, ketone body synthesis, and the urea cycle [59].

When three generations of women with the same maternally inherited mtDNA sequence were studied, an age-associated decline in the skeletal muscle content of SIRT3 and mtDNA copy numbers as well as an increase in mitochondrial protein acetylation were observed [60]. This result was confirmed on male subjects also [61]. The increased level of protein acetylation is due to increased activity of the mitochondrial acetylase GCN5 and the decreased activity of SIRT3. However, fasting or other metabolic challenges increase the activity of SIRT3, which deacetylates, and therefore activates, the enzyme responsible for formation of acetyl-CoA from acetate [acetyl-CoA-synthase (AceCS) [62]. AcsCS is then converted to acetyl-CoA and utilized in the citric acid cycle. Among the wide range of deacetylating targets of SIRT3, we can find p53, p300, Ku70, mitochondrial ribosomal protein L10 and metabolic regulators like ornithine transcarbamoylase, succinate dehydrogenase, and FOXO3 [58].

Because exercise brings a metabolic challenge to most of the organs, either by increasing or decreasing blood and energy supplies, SIRT3 is a potential mitochondrial protein, which could mediate exercise-associated adaptation. SIRT3 levels are increased in those organs which have a higher metabolic rate, including liver, kidney, and heart [63] and are readily up-regulated by caloric restriction [64] and exercise training [65]. Hence, the levels of SIRT-3 are elevated in skeletal muscle of trained subjects $[66,67]$. Besides controlling lipid and carbohydrate metabolism, SIRT3 also deacetylates and activates SOD2, and isocitrate dehydrogenase 2, an enzyme in the TCA cycle that helps to replenish the mitochondrial pool of NADPH [63]. SIRT3 levels are increased with exercise training in the hippocampus, skeletal muscle, liver and heart [67-71].

Based on current knowledge, it cannot be completely ruled out that mitochondrial content, dynamics and function are linked to a systemic 
exercise-associated adaptation, which includes a decreased rate of mortality and enhanced levels of antioxidant and oxidative damage repair systems (Fig. 1).

\section{Exercise, aging and main housekeeping systems}

The health benefit effects of exercise and the mortality decreasing effects are generally seen at about age 60 , while in younger healthy populations, higher level of cardiovascular fitness is not associated with a decreased rate of mortality [72,73]. Aging is associated with a decreased ability to maintain homeostasis, and decreased physiological functions, which includes increased production of ROS and decreased levels of anti-oxidant housekeeping enzymes such as the proteasome and Lon protease [74]. The effects of aging are systemic and the effects of physical exercise are also systemic. The fact that the effects of physical exercise are systemic is a key component for offsetting or prolonging the systemic effects of aging.

The aging process itself is clearly linked to life styles such as nutrition, health care education and physical activity. It is suggested that these environmental factors account for $70 \%$ of the variation in life span [75] and the avoidance of the risk factors that cause many diseases [76]. As stated earlier, the level of aerobic capacity, $\mathrm{VO}_{2} \mathrm{max}$, is inversely related to mortality [12].

Aging, especially in the last quarter of an average life span, is associated with an increased accumulation of oxidative damage [7] and a decrease in organ function. An alternative theory suggests that the ageassociated loss in stem cell number and/or activity over time causes a decline in organ function. However, according to our current understanding, the mechanism behind the aging of stem cell populations appears to be similar for other cell types. One of the major factors is oxidative stress [77]. Oxidative stress is often evaluated by oxidative damage markers, the levels of which are dependent on the production of ROS, the rate of ROS elimination by antioxidant systems, and the efficiency of the oxidative damage repair systems, which often are cumulatively called the housekeeping system.

\subsection{Proteasome system}

The proteasome system is the primary protein degradation pathway in the cell. It is responsible for removal of damaged e.g. oxidatively modified proteins, and those of short-lived proteins. Although the exact molecular mechanism is unclear, an age-related accumulation of damaged proteins favors the aging processes and the development of diseases. Johnson and co-workers studied the effects of endurance training on protein degradation in human subjects using mass spectrometry to quantify semitryptic peptides and fluorogenic substrates for peptidylglutamyl-like activity of the 26S Proteasome [69]. Results showed that eight weeks of exercise training increased total and mitochondrial protein semitryptic peptides in young humans while older participants exhibited a decline. Similar data were obtained for proteasome assays [69]. The authors suggested that young subjects cope with exercise-induced oxidative challenge with enhanced housekeeping, while older subjects increased the buffering capacity against ROS by enhanced antioxidant system activity [69]. In another human study, where the acute effects of resistance exercise were studied, the proteasome system was activated in the skeletal muscle of old subjects (72 \pm 2 years) [78]. It is important to note that the type of exercise (acute, chronic, endurance, resistance, etc.) and the type of measured proteasome activity (chymotrypsin-like, trypsin-like, caspase-like) had a significant impact on the results.

Most studies on animals used aerobic endurance exercise to investigate the response of proteasome to physical training. We have reported that exercise training increased the activities of the proteasome in skeletal muscle [79] and the brain [80] of young and old trained rats. The fact that exercise increases the activity of the proteasome in the brain could be very important since it was shown that the proteasome can degrade beta-amyloids [81], which could partly explain why regular exercise decreases the incidence of Alzheimer Disease [82].

The increases in proteasome activity might reflect a faster rate of protein turnover, and/or better remodeling after exercise-associated micro injuries. On the other hand, when aged rats were subjected to immobilization, the associated increase in proteasome activity was attenuated by aerobic exercise [83], suggesting that exercise can enhance recovery after atrophic stimulus. Ziaaldini and co-workers [84] also reported that six weeks of exercise training at $60 \%$ of $\mathrm{VO}_{2}$ max reversed the age-associated activation of catabolic and apoptotic pathways and increased anabolic signaling. These results suggest that the age-associated loss of muscle mass and cachexia could be due to the orchestrated down-regulation of anabolic and up-regulation of catabolic and pro-apoptotic processes [84]. Muscle-RING-finger protein 1 (MuRF-1) is an ubiquitin ligase and is considered to be linked to proteolytic degradation. When MuRF-1 levels were checked in old and young women after a single bout of resistance exercise, data showed that, in both age groups, the MuRF-1 increased within four hours [85]. MuRF-1 adds ubiquitin to target proteins like NRF1, which results in protein degradation by proteasome complex [86]. It is important to note that the proteasome, which targets K48- linked ubiquitin chains [87], is also involved in the regulation of proteins for many cellular processes, including cell cycle and transcriptional regulation and even in DNA repair [88]. The proteasome degrades p53 when it is ubiquitinated by oncoprotein MDM2, which behaves as an E3 ubiquitin ligase [89]. The results of a recent study that used a hindlimb unloading model on mice with a supplementation of allopurinol, which attenuated muscle wasting, suggests that the proteasome system is indeed an appropriate target to prevent muscle atrophy [90].

\subsection{Lon protease}

Lon is localized in the mitochondria and is an important factor in quality control of mitochondrial proteins. The levels/activities of Lon protein decline with age, which could result in the accumulation of oxidized or dysfunctional proteins in the mitochondria [91,92] leading to the loss of mitochondrial function. Hindlimb immobilization led to down- regulated Mfn and Fis-1 proteins along with Lon protease levels in murine gastrocnemius muscle [93]. On the other hand, we have shown that regular exercise can prevent the age-related decline in Lon in skeletal muscle of rats and, therefore, rejuvenate proper quality control of proteins in older cells [39]. Moreover, it has also been shown that aerobic training- responder rats have higher levels of Lon protease than non-responders after exercise training, suggesting better mitochondrial housekeeping [94]. These results suggest that exercise training results in up-regulated housekeeping in the mitochondria of skeletal muscle.

\section{3. $p 53$}

The transcription factor p53 is involved in complex regulatory systems to protect the genome and cope with a variety of stressors. In response to DNA damage, members of the p53 family are stabilized via post-translational modifications and are functionally involved in the initiation of various DNA damage-driven cellular responses that eventually determine the fate of cells, by modulating the expression of factors key to cell proliferation, the process of DNA repair, and as a result, the aging process [95]. In a study where progeroid aging was mimicked by an inherent defect in mitochondrial polymerase gamma (POLG1) proofreading function, results showed that five months of endurance exercise induced systemic mitochondrial biogenesis, prevented mtDNA depletion and mutations, increased mitochondrial oxidative capacity and respiratory chain assembly, restored mitochondrial morphology, and blunted pathological levels of apoptosis in multiple tissues of mtDNA mutator mice [96]. Endurance exercise completely 
rescued mtDNA depletion in skeletal muscle, heart, and liver of DNA polymerase gamma (PolG) mice. When oxidative damage to POLG1 mutator mice was studied, it was noted that mitochondria from tissues of mutator mice demonstrated a substantial increase in $\mathrm{H}_{2} \mathrm{O}_{2}$ production, along with elevated protein carbonyls and 4-hydroxynonenal (4HNE) content, which was attenuated by exercise training [97]. Moreover, endurance exercise diminished telomere erosion and down-regulated aberrant p53 signaling and pathological levels of apoptosis in PolG mice [97]. Furthermore, it was shown that tumor suppressor protein p53 translocates to mitochondria and facilitates mtDNA mutation repair and mitochondrial biogenesis in response to endurance exercise. p53 is one of the most well studied proteins, due to its role in DNA repair, but p53 may regulate contractile-induced increases in mitochondrial content via modulating TFAM activity, given that p53 KO mice display reduced skeletal muscle mitochondrial DNA, TFAM messenger RNA and protein levels [98]. Indeed, p53 ablation results in significant decreases in exercise capacity (in the magnitude of 50\%) for treadmill running [98]. p53 is one of the prime targets of SIRT1 which is readily activated by physical exercise, probably through direct phosphorylation by AMPK [99], which leads to the deacetylation/inactivation of p53 [57,100].

\subsection{Autophagy}

Autophagy and mitophagy are important parts of cellular housekeeping. Autophagy is a catabolic process, which encompasses the different routes that cells use to deliver cytoplasmic substrates to lysosomes for degradation. Autophagy is a cytoprotective process in which non-nuclear (cytoplasmic) parts of the cell can be renewed to meet the bioenergetic demand of the cell in conditions of dwindling external or internal resources [101]. Indeed, autophagy is also an important regulator of the homeostasis of mitochondria. Dysfunctional mitochondria that have lost their membrane potential and are more likely to release ROS and be targets of autophagy, are replaced by fit mitochondria via phosphorylation reactions mediated by the kinase PINK1 and subsequent ubiquitination of mitochondrial membrane proteins by the E3 ligase Parkin [101].

Ablation of PGC1 $\alpha$ has been shown to result in a $40 \%$ decrease in mitochondrial content, as well as a $25 \%$ decline in running performance, while a single bout of exercise induced increased transcript levels of the autophagy factors LC3B and p62 in wild but not in KO mice [102]. ROS are important regulators of autophagy, and the cross talk appears to be bidirectional [103], meaning that ROS production can prevent a positive feedback loop whereby increased autophagy is stimulated by oxidative stress, resulting in further increases in ROS and autophagy [103]. Autophagy can be linked to aging, because longevity can be modulated via mTOR suppression [101]. Physical exercise can act through AMPK and SIRT1 activation pathways to attenuate the aging process via autophagy [104].

\subsection{Mitophagy}

Significant damage to mitochondria can result in the ubiquitination and proteasomal degradation of Mfn1 and Mfn2 and subsequent mitophagy [105]. AMPK has been reported to regulate autophagy and mitophagy by activating unc-51- like autophagy activating kinase 1 (ULK1) [106]. Endurance exercise at the intensity of $70 \%$ of $\mathrm{VO}_{2} \mathrm{max}$ for 30 min significantly increased the phosphorylation of ULK1 ${ }^{\text {Ser757 }}$ in the vastus lateralis muscle of young subjects [107]. In studies on mice, the increase in mitophagy markers of BNIP3 and Parkin suggest that mitophagy was increased after 90 min running with $10 \mathrm{~m} / \mathrm{min}$ speed in animals who run at fasted conditions compared to fed control groups [108]. AMPK and muscle-specific knockouts of Ulk1 transgenic mice have been used to study the effects of exercise on muscle mitophagy [109]. Data showed that during exercise, AMPK phosphorylates Ulk1, which is required for the resolution of exercise-induced mitochondrial damage through mitophagy during recovery [109]. Deletion of the Ulk1 gene in skeletal muscle inhibits mitophagy without affecting lysosome formation in response to exercise, which suggests a novel role for Ulk1 in targeting lysosomes to damaged/dysfunctional mitochondria that has implications for metabolic adaptations to exercise training. The investigators used MitoTimer and 4HNE to assess oxidative stress, and found increased generation of ROS, three and six hours after exercise. These findings suggest that during the recovery phase, the continued oxidative phosphorylation and sudden reduction of ATP consumption dominate the excessive production of mitochondrial ROS [109].

\subsection{Base excision repair}

Regular exercise has been shown to decrease the incidence of mortality in cancer and it is suggested that this could be partly due to the fact, that exercise increases the activity of DNA repair enzymes [110]. Oxidative DNA base damage is primarily repaired via the base excision repair pathway that is initiated by DNA glycosylases. The most abundant DNA base lesion is 7, 8-dihydro-8-oxoguanine (8-oxoG) due to the fact that guanine has the lowest redox potential among the four nucleobases [111]. 8-OxoG is repaired primarily by 8-oxoguanine DNA glycosylase1 (OGG1), which recognizes and excises 8-oxoG and ringfragmented purines, including 2,6-diamino-4-hydroxy-5-formamidopyrimidine (FapyG). OGG1 is not just the most well studied repair enzyme, but also its role in cleaving 8-oxoG links it to cellular signaling [112], metabolism [113], inflammation [114] and epigenetics [114-116]. Interestingly, polymorphisms in the OGG1 gene is associated with the incidence of type II diabetes in human cohorts [117].

We have reported that endurance exercise at an intensity of $70 \%$ of $\mathrm{VO}_{2}$ max resulted in increased activity of OGG1 and decreased accumulation of 8-oxoG in skeletal muscle [118] and liver of rats [119] and exercise attenuated the age-associated loss in the activity of OGG1. The activity of OGG1 is greater in slow fibers than fast fibers [38] and the activity is increased in the nucleus and decreased in mitochondria as a result of exercise training [38,119]. When ROS production, 8-oxoG, and OGG1 were studied in high and low running capacity rats, data revealed that high running capacity was associated with an increased production of $\mathrm{H}_{2} \mathrm{O}_{2}$, decreased levels of 8-oxoG and no difference in the protein level of OGG1. This suggested that genetic segregation for high running capacity has generated a molecular network of cellular adaptations, facilitating a superior response to oxidative stress [120].

Both acute and regular exercise increases the activity of OGG1 in human skeletal muscle $[67,121]$, when acetylated by p300 [122] and deacetylated by SIRT1 [123].

Recently, studies on OGG1 suggest that OGG1 is linked to regulation of mitochondrial dynamics in both in vivo and in vitro models. Cells overexpressing OGG1 had increased membrane potential and decreased mitochondrial fragmentation [124]. The protein levels of fission and apoptotic factors such as DRP1, FIS1, cytoplasmic cytochrome c, were decreased in adenoviral OGG1 overexpressed cells. These observations suggest that OGG1 overexpression may be an important mechanism to protect cardiac cells against oxidative stress damage [124]. Moreover, it turned out that ablation of OGG1 is associated with metabolic syndrome, insulin resistance, increases in muscle lipid droplets, and altered fat metabolism $[113,125]$. These data suggest that the enzyme, which repairs the most abundant oxidative modification of DNA, 8-oxoG, has a complex regulatory function, and indicates a need to re-evaluate the role of exercise-induced activity of OGG1. Therefore, based on the data obtained from OGG1 knock-out models, it cannot be excluded that the well-known preventive or curative effects of regular exercise on metabolic syndrome, obesity, and insulin resistance at the cellular level, are at least in part, modulated by the stimulating effects of exercise on OGG1. Exercise indeed, increases OGG1 activity in skeletal muscle [126], liver [119], and brain [123]. 


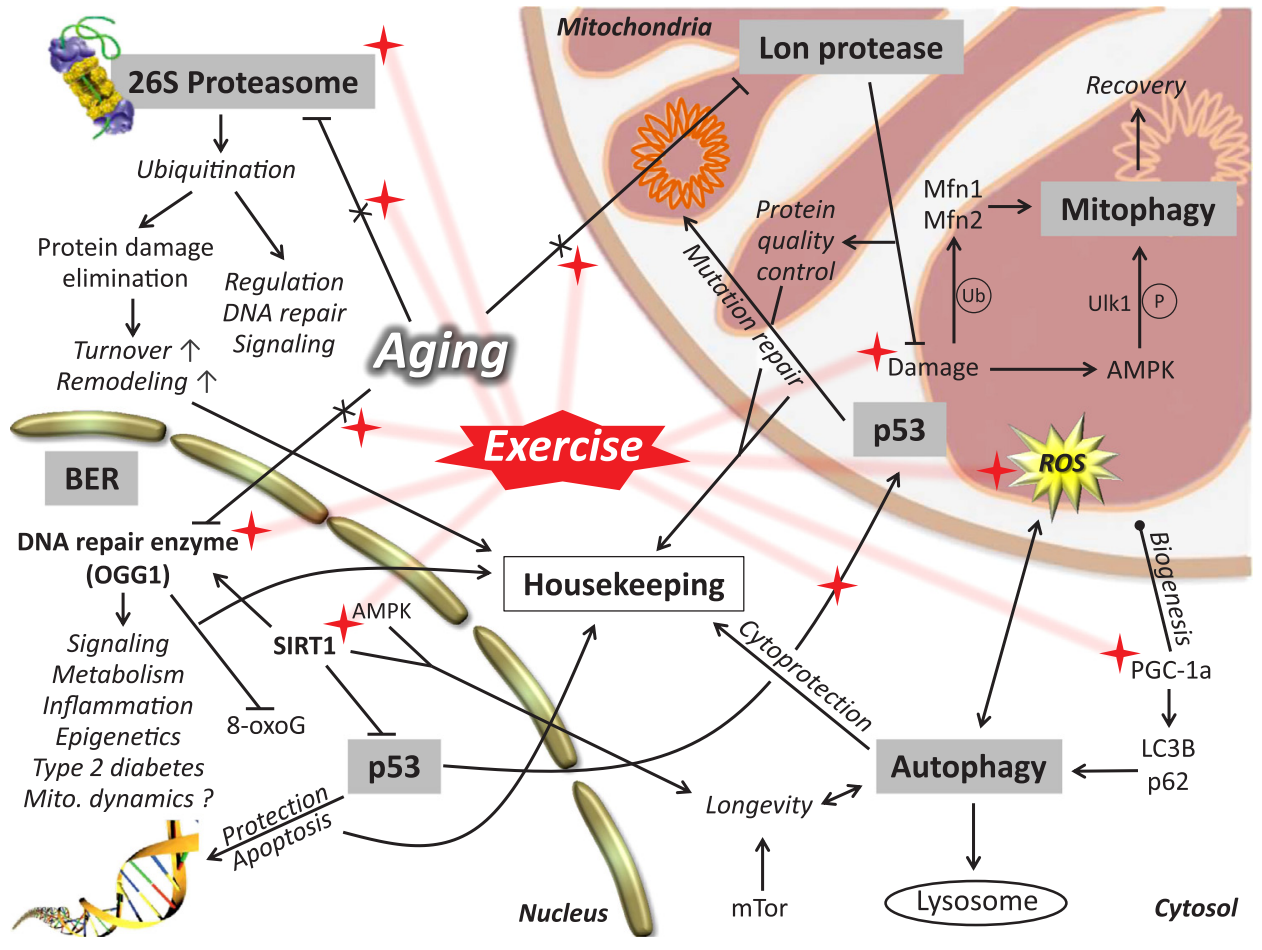

Fig. 2. Regular exercise increases the efficiency of cellular housekeeping. Aging is often associated with decline of the cellular housekeeping system resulting in accumulation of damage and impaired function. The main housekeeping systems, such as autophagy, mitophagy, proteasome, Lon protease, p53 and the base excision repair are all benefically effected by regular exercise. The enhanced housekeeping is essential to improved cellular and organ function as well as to decelerating the aging process.

\section{Conclusion}

Elevation of maximal oxygen uptake is directly correlated with decreased mortality, and beneficially effects organ functions, including the efficiency of the antioxidant and repair systems. It appears that regular exercise increases mitochondrial biogenesis, dynamics and SIRT3 activity, in all the measured organs. However, the systemic effects of exercise are not dependent solely on a single mitochondrial factor. Despite the fact that regular exercise attenuates most age-related deteriorations in mitochondrial function or dynamics, we cannot narrow the signaling pathways into a single protein or cellular pathway. Regular exercise increases the activity of cellular housekeeping, for which efficiency declines significantly with age and includes the proteasome complex, Lon protease, autophagy, mitophagy and the activity DNA repair enzymes involved in ROS mediated damage (Fig. 2). Regular exercise increases the activity of OGG1, which is involved in DNA repair, cellular signaling and epigenetics, demonstrating the complex effects of exercise on cellular function and attenuation of the aging process. Overall, it seems that redox signaling pathways are very important in the systemic health promoting effects of physical exercise, and these pathways are multi branched.

\section{Acknowledgements}

This study was supported by National Scientific Research Foundation, Hungary (OTKA 112810) and National Excellence Program Hungary (126823) awarded to Z.R. Authors acknowledge the assistance of Professor A.W. Taylor in the preparation of the manuscript. E.K and L.B. were supported by GINOP-2.3.2-15-2016-00062. P.A. was supported by 20391-3/2018/FEKUSTRAT, Hungarian grant. For editing this manuscript, we thank Sherry L. Haller, PhD, English Editor, Research Development Specialist, The University of Texas Medical Branch, Galveston.

\section{References}

[1] Z. Radak, H.Y. Chung, S. Goto, Systemic adaptation to oxidative challenge induced by regular exercise, Free Radic. Biol. Med. 44 (2008) 153-159.

[2] G. Lacza, Z. Radak, Is physical activity an elixir? Orv. Hetil. 154 (2013) 764-768.
[3] M.R. Carnethon, M. Gulati, P. Greenland, Prevalence and cardiovascular disease correlates of low cardiorespiratory fitness in adolescents and adults, JAMA 294 (2005) 2981-2988.

[4] L.B. Andersen, Physical activity and physical fitness as protection against premature disease or death, Scand. J. Med. Sci. Sports 5 (1995) 318-328.

[5] D.M. Bramble, D.E. Lieberman, Endurance running and the evolution of Homo, Nature 432 (2004) 345-352.

[6] D.E. Lieberman, D.M. Bramble, The evolution of marathon running: capabilities in humans, Sports Med. 37 (2007) 288-290.

[7] Z. Radak, Z. Zhao, E. Koltai, H. Ohno, M. Atalay, Oxygen consumption and usage during physical exercise: the balance between oxidative stress and ROS-dependent adaptive signaling, Antioxid. Redox Signal. 18 (2013) 1208-1246.

[8] J. Denham, F.Z. Marques, B.J. O'Brien, F.J. Charchar, Exercise: putting action into our epigenome, Sports Med. 44 (2014) 189-209.

[9] J. Rosado-Perez, V.M. Mendoza-Nunez, Relationship between aerobic capacity with oxidative stress and inflammation biomarkers in the blood of older mexican urban-dwelling population, Dose Response 16 (2018) (1559325818773000).

[10] Z. Radak, H.Y. Chung, E. Koltai, A.W. Taylor, S. Goto, Exercise, oxidative stress and hormesis, Ageing Res. Rev. 7 (2008) 34-42.

[11] A. Solomon, K. Borodulin, T. Ngandu, M. Kivipelto, T. Laatikainen, J. Kulmala, Self-rated physical fitness and estimated maximal oxygen uptake in relation to all cause and cause-specific mortality, Scand. J. Med. Sci. Sports (2017).

[12] M.T. Jensen, A. Holtermann, H. Bay, F. Gyntelberg, Cardiorespiratory fitness and death from cancer: a 42-year follow-up from the Copenhagen Male Study, Br. J. Sports Med. 51 (2017) 1364-1369.

[13] L.H. Galant, L.A. Forgiarini Junior, A.S. Dias, C.A. Marroni, Maximum oxygen consumption predicts mortality in patients with alcoholic cirrhosis, Hepatogastroenterology 60 (2013) 1127-1130.

[14] A. Bohm, C. Weigert, H. Staiger, H.U. Haring, Exercise and diabetes: relevance and causes for response variability, Endocrine 51 (2016) 390-401.

[15] M. Park, Y. Ko, S.H. Song, S. Kim, H.J. Yoon, Association of low aerobic fitness with hyperfiltration and albuminuria in men, Med. Sci. Sports Exerc. 45 (2013) 217-223.

[16] C. Totsikas, J. Rohm, K. Kantartzis, C. Thamer, K. Rittig, J. Machann, F. Schick, J. Hansel, A. Niess, A. Fritsche, H.U. Haring, N. Stefan, Cardiorespiratory fitness determines the reduction in blood pressure and insulin resistance during lifestyle intervention, J. Hypertens. 29 (2011) 1220-1227.

[17] H. Watz, F. Pitta, C.L. Rochester, J. Garcia-Aymerich, R. ZuWallack, T. Troosters, A.W. Vaes, M.A. Puhan, M. Jehn, M.I. Polkey, I. Vogiatzis, E.M. Clini, M. Toth, E. Gimeno-Santos, B. Waschki, C. Esteban, M. Hayot, R. Casaburi, J. Porszasz, E. McAuley, S.J. Singh, D. Langer, E.F. Wouters, H. Magnussen, M.A. Spruit, An official European Respiratory Society statement on physical activity in COPD, Eur. Respir. J. 44 (2014) 1521-1537.

[18] Z. Radak, K. Suzuki, M. Higuchi, L. Balogh, I. Boldogh, E. Koltai, Physical exercise, reactive oxygen species and neuroprotection, Free Radic. Biol. Med. 98 (2016) 187-196.

[19] J.A. Timmons, Variability in training-induced skeletal muscle adaptation, J. Appl. Physiol. 110 (2011) 846-853.

[20] R.A. Jacobs, C. Lundby, Mitochondria express enhanced quality as well as quantity in association with aerobic fitness across recreationally active individuals up to 
elite athletes, J. Appl. Physiol. 114 (2013) 344-350.

21] K.J. Davies, L. Packer, G.A. Brooks, Biochemical adaptation of mitochondria, muscle, and whole-animal respiration to endurance training, Arch. Biochem. Biophys. 209 (1981) 539-554.

[22] N.B. Vollaard, D. Constantin-Teodosiu, K. Fredriksson, O. Rooyackers, E. Jansson, P.L. Greenhaff, J.A. Timmons, C.J. Sundberg, Systematic analysis of adaptations in aerobic capacity and submaximal energy metabolism provides a unique insight into determinants of human aerobic performance, J. Appl. Physiol. 106 (2009) $1479-1486$.

[23] M.J. Laye, M.B. Nielsen, L.S. Hansen, T. Knudsen, B.K. Pedersen, Physical activity enhances metabolic fitness independently of cardiorespiratory fitness in marathon runners, Dis. Markers 2015 (2015) 806418.

[24] D. Martinez-Redondo, A. Marcuello, J.A. Casajus, I. Ara, Y. Dahmani, J. Montoya, E. Ruiz-Pesini, M.J. Lopez-Perez, C. Diez-Sanchez, Human mitochondrial haplogroup H: the highest VO2max consumer-is it a paradox? Mitochondrion 10 (2010) 102-107.

[25] V. Macaulay, M. Richards, E. Hickey, E. Vega, F. Cruciani, V. Guida, R. Scozzari, B. Bonne-Tamir, B. Sykes, A. Torroni, The emerging tree of West Eurasian mtDNAs: a synthesis of control-region sequences and RFLPs, Am. J. Hum. Genet. 64 (1999) 232-249.

[26] E. Dominguez-Garrido, D. Martinez-Redondo, C. Martin-Ruiz, A. Gomez-Duran, E. Ruiz-Pesini, P. Madero, M. Tamparillas, J. Montoya, T. von Zglinicki, C. DiezSanchez, M.J. Lopez-Perez, Association of mitochondrial haplogroup J and mtDNA oxidative damage in two different North Spain elderly populations, Biogerontology 10 (2009) 435-442.

[27] J.O. Holloszy, Biochemical adaptations in muscle. Effects of exercise on mitochondrial oxygen uptake and respiratory enzyme activity in skeletal muscle, J. Biol. Chem. 242 (1967) 2278-2282.

[28] A. Boveris, A. Navarro, Systemic and mitochondrial adaptive responses to moderate exercise in rodents, Free Radic. Biol. Med. 44 (2008) 224-229.

[29] K. Marosi, Z. Bori, N. Hart, L. Sarga, E. Koltai, Z. Radak, C. Nyakas, Long-term exercise treatment reduces oxidative stress in the hippocampus of aging rats, Neuroscience 226 (2012) 21-28.

[30] A.M. Joseph, L.M. Nguyen, A.E. Welter, J.M. Dominguez 2nd, B.J. Behnke, P.J. Adhihetty, Mitochondrial adaptations evoked with exercise are associated with a reduction in age-induced testicular atrophy in Fischer-344 rats, Biogerontology 15 (2014) 517-534.

[31] P. Puigserver, B.M. Spiegelman, Peroxisome proliferator-activated receptorgamma coactivator 1 alpha (PGC-1 alpha): transcriptional coactivator and metabolic regulator, Endocr. Rev. 24 (2003) 78-90.

[32] A.T. Erlich, L.D. Tryon, M.J. Crilly, J.M. Memme, Z.S.M. Moosavi, A.N. Oliveira, K. Beyfuss, D.A. Hood, Function of specialized regulatory proteins and signaling pathways in exercise-induced muscle mitochondrial biogenesis, Integr. Med. Res 5 (2016) 187-197.

[33] A. Philp, S. Schenk, Unraveling the complexities of SIRT1-mediated mitochondrial regulation in skeletal muscle, Exerc. Sport Sci. Rev. 41 (2013) 174-181.

[34] R.C. Scarpulla, Metabolic control of mitochondrial biogenesis through the PGC-1 family regulatory network, Biochim. Biophys. Acta (2011) 1269-1278.

[35] L. Leick, S.S. Lyngby, J.F. Wojtaszewski, H. Pilegaard, PGC-1alpha is required for training-induced prevention of age-associated decline in mitochondrial enzymes in mouse skeletal muscle, Exp. Gerontol. 45 (2010) 336-342.

[36] C. Handschin, S. Chin, P. Li, F. Liu, E. Maratos-Flier, N.K. Lebrasseur, Z. Yan, B.M. Spiegelman, Skeletal muscle fiber-type switching, exercise intolerance, and myopathy in PGC-1alpha muscle-specific knock-out animals, J. Biol. Chem. 282 (2007) 30014-30021.

[37] D. Marmolino, M. Manto, F. Acquaviva, P. Vergara, A. Ravella, A. Monticelli, M. Pandolfo, PGC-1alpha down-regulation affects the antioxidant response in Friedreich's ataxia, PLoS One 5 (2010) e10025.

[38] Z. Radak, S. Kumagai, H. Nakamoto, S. Goto, 8-Oxoguanosine and uracil repair of nuclear and mitochondrial DNA in red and white skeletal muscle of exercisetrained old rats, J. Appl. Physiol. 102 (2007) 1696-1701.

[39] E. Koltai, N. Hart, A.W. Taylor, S. Goto, J.K. Ngo, K.J. Davies, Z. Radak, Ageassociated declines in mitochondrial biogenesis and protein quality control factors are minimized by exercise training, Am. J. Physiol. Regul. Integr. Comp. Physiol. 303 (2012) R127-R134.

[40] C.G. Perry, J. Lally, G.P. Holloway, G.J. Heigenhauser, A. Bonen, L.L. Spriet, Repeated transient mRNA bursts precede increases in transcriptional and mitochondrial proteins during training in human skeletal muscle, J. Physiol. 588 (2010) 4795-4810.

[41] D. Bach, S. Pich, F.X. Soriano, N. Vega, B. Baumgartner, J. Oriola, J.R. Daugaard, J. Lloberas, M. Camps, J.R. Zierath, R. Rabasa-Lhoret, H. Wallberg-Henriksson, M. Laville, M. Palacin, H. Vidal, F. Rivera, M. Brand, A. Zorzano, Mitofusin-2 determines mitochondrial network architecture and mitochondrial metabolism. A novel regulatory mechanism altered in obesity, J. Biol. Chem. 278 (2003) 17190-17197.

[42] A. Vital, C. Vital, Mitochondria and peripheral neuropathies, J. Neuropathol. Exp. Neurol. 71 (2012) 1036-1046.

[43] Y. Arribat, N.T. Broskey, C. Greggio, M. Boutant, S. Conde Alonso, S.S. Kulkarni, S. Lagarrigue, E.A. Carnero, C. Besson, C. Canto, F. Amati, Distinct patterns of skeletal muscle mitochondria fusion, fission and mitophagy upon duration of exercise training, Acta Physiol. (2018) e13179.

[44] A.K. Meinild Lundby, R.A. Jacobs, S. Gehrig, J. de Leur, M. Hauser, T.C. Bonne, D. Fluck, S. Dandanell, N. Kirk, A. Kaech, U. Ziegler, S. Larsen, C. Lundby, Exercise training increases skeletal muscle mitochondrial volume density by enlargement of existing mitochondria and not de novo biogenesis, Acta Physiol. 222 (2018).

45] V.L. Wyckelsma, I. Levinger, M.J. McKenna, L.E. Formosa, M.T. Ryan,
A.C. Petersen, M.J. Anderson, R.M. Murphy, Preservation of skeletal muscle mitochondrial content in older adults: relationship between mitochondria, fibre type and high-intensity exercise training, J. Physiol. 595 (2017) 3345-3359.

[46] S. Cipolat, O. Martins de Brito, B. Dal Zilio, L. Scorrano, OPA1 requires mitofusin 1 to promote mitochondrial fusion, Proc. Natl. Acad. Sci. USA 101 (2004) $15927-15932$.

[47] S. Zampieri, C. Mammucari, V. Romanello, L. Barberi, L. Pietrangelo, A. Fusella, S. Mosole, G. Gherardi, C. Hofer, S. Lofler, N. Sarabon, J. Cvecka, M. Krenn, U. Carraro, H. Kern, F. Protasi, A. Musaro, M. Sandri, R. Rizzuto, Physical exercise in aging human skeletal muscle increases mitochondrial calcium uniporter expression levels and affects mitochondria dynamics, Physiol. Rep. 4 (2016).

[48] Y. Kitaoka, K. Nakazato, R. Ogasawara, Combined effects of resistance training and calorie restriction on mitochondrial fusion and fission proteins in rat skeletal muscle, J. Appl. Physiol. 121 (2016) 806-810.

[49] C. Liao, N. Ashley, A. Diot, K. Morten, K. Phadwal, A. Williams, I. Fearnley, L. Rosser, J. Lowndes, C. Fratter, D.J. Ferguson, L. Vay, G. Quaghebeur, I. Moroni, S. Bianchi, C. Lamperti, S.M. Downes, K.S. Sitarz, P.J. Flannery, J. Carver, E. Dombi, D. East, M. Laura, M.M. Reilly, H. Mortiboys, R. Prevo, M. Campanella, M.J. Daniels, M. Zeviani, P. Yu-Wai-Man, A.K. Simon, M. Votruba, J. Poulton, Dysregulated mitophagy and mitochondrial organization in optic atrophy due to OPA1 mutations, Neurology 88 (2017) 131-142.

[50] J.F. Halling, S. Ringholm, J. Olesen, C. Prats, H. Pilegaard, Exercise training protects against aging-induced mitochondrial fragmentation in mouse skeletal muscle in a PGC-1alpha dependent manner, Exp. Gerontol. 96 (2017) 1-6.

[51] Z. Bori, Z. Zhao, E. Koltai, I.G. Fatouros, A.Z. Jamurtas, I.I. Douroudos, G. Terzis, A. Chatzinikolaou, A. Sovatzidis, D. Draganidis, I. Boldogh, Z. Radak, The effects of aging, physical training, and a single bout of exercise on mitochondrial protein expression in human skeletal muscle, Exp. Gerontol. 47 (2012) 417-424.

[52] A.M. Gusdon, J. Callio, G. Distefano, R.M. O'Doherty, B.H. Goodpaster, P.M. Coen, C.T. Chu, Exercise increases mitochondrial complex I activity and DRP1 expression in the brains of aged mice, Exp. Gerontol. 90 (2017) 1-13.

[53] L. Luo, J.R. Dai, S.S. Guo, A.M. Lu, X.F. Gao, Y.R. Gu, X.F. Zhang, H.D. Xu, Y. Wang, Z. Zhu, L.J. Wood, Z.H. Qin, Lysosomal proteolysis is associated with exercise-induced improvement of mitochondrial quality control in aged hippocampus, J. Gerontol. A Biol. Sci. Med. Sci. 72 (2017) 1342-1351.

[54] E. Chung, H.E. Joiner, T. Skelton, K.D. Looten, M. Manczak, P.H. Reddy, Materna exercise upregulates mitochondrial gene expression and increases enzyme activity of fetal mouse hearts, Physiol. Rep. 5 (2017).

[55] R. Boushel, E. Gnaiger, J.A. Calbet, J. Gonzalez-Alonso, C. Wright-Paradis, H. Sondergaard, I. Ara, J.W. Helge, B. Saltin, Muscle mitochondrial capacity exceeds maximal oxygen delivery in humans, Mitochondrion 11 (2011) 303-307.

[56] D.A. Hood, L.D. Tryon, H.N. Carter, Y. Kim, C.C. Chen, Unravelling the mechanisms regulating muscle mitochondrial biogenesis, Biochem. J. 473 (2016) $2295-2314$

[57] Z. Radak, E. Koltai, A.W. Taylor, M. Higuchi, S. Kumagai, H. Ohno, S. Goto, I. Boldogh, Redox-regulating sirtuins in aging, caloric restriction, and exercise, Free Radic. Biol. Med. 58 (2013) 87-97.

[58] R. Nogueiras, K.M. Habegger, N. Chaudhary, B. Finan, A.S. Banks, M.O. Dietrich, T.L. Horvath, D.A. Sinclair, P.T. Pfluger, M.H. Tschop, Sirtuin 1 and sirtuin 3: physiological modulators of metabolism, Physiol. Rev. 92 (2012) 1479-1514.

[59] A.S. Hebert, K.E. Dittenhafer-Reed, W. Yu, D.J. Bailey, E.S. Selen, M.D. Boersma, J.J. Carson, M. Tonelli, A.J. Balloon, A.J. Higbee, M.S. Westphall, D.J. Pagliarini, T.A. Prolla, F. Assadi-Porter, S. Roy, J.M. Denu, J.J. Coon, Calorie restriction and SIRT3 trigger global reprogramming of the mitochondrial protein acetylome, Mol. Cell 49 (2013) 186-199.

[60] S.L. Hebert, P. Marquet-de Rouge, I.R. Lanza, S.K. McCrady-Spitzer, J.A. Levine, S. Middha, R.E. Carter, K.A. Klaus, T.M. Therneau, E.W. Highsmith, K.S. Nair, Mitochondrial aging and physical decline: insights from three generations of women, J. Gerontol. A Biol. Sci. Med. Sci. 70 (2015) 1409-1417.

[61] A.M. Joseph, P.J. Adhihetty, T.W. Buford, S.E. Wohlgemuth, H.A. Lees, L.M. Nguyen, J.M. Aranda, B.D. Sandesara, M. Pahor, T.M. Manini, E. Marzetti, C. Leeuwenburgh, The impact of aging on mitochondrial function and biogenesis pathways in skeletal muscle of sedentary high- and low-functioning elderly individuals, Aging Cell 11 (2012) 801-809.

[62] B. Schwer, J. Bunkenborg, R.O. Verdin, J.S. Andersen, E. Verdin, Reversible lysine acetylation controls the activity of the mitochondrial enzyme acetyl-CoA synthetase 2, Proc. Natl. Acad. Sci. USA 103 (2006) 10224-10229.

[63] J.C. Newman, W. He, E. Verdin, Mitochondrial protein acylation and intermediary metabolism: regulation by sirtuins and implications for metabolic disease, J. Biol. Chem. 287 (2012) 42436-42443.

[64] C. Han, S. Someya, Maintaining good hearing: calorie restriction, Sirt3, and glutathione, Exp. Gerontol. 48 (2013) 1091-1095.

[65] A.T. White, S. Schenk, NAD(+)/NADH and skeletal muscle mitochondrial adaptations to exercise, Am. J. Physiol. Endocrinol. Metab. 303 (2012) E308-E321.

[66] I.R. Lanza, D.K. Short, K.R. Short, S. Raghavakaimal, R. Basu, M.J. Joyner, J.P. McConnell, K.S. Nair, Endurance exercise as a countermeasure for aging, Diabetes 57 (2008) 2933-2942.

[67] Z. Radak, Z. Bori, E. Koltai, I.G. Fatouros, A.Z. Jamurtas, I.I. Douroudos, G. Terzis, M.G. Nikolaidis, A. Chatzinikolaou, A. Sovatzidis, S. Kumagai, H. Naito, I. Boldogh, Age-dependent changes in 8-oxoguanine-DNA glycosylase activity are modulated by adaptive responses to physical exercise in human skeletal muscle, Free Radic. Biol. Med. 51 (2011) 417-423.

[68] A. Cheng, Y. Yang, Y. Zhou, C. Maharana, D. Lu, W. Peng, Y. Liu, R. Wan, K. Marosi, M. Misiak, V.A. Bohr, M.P. Mattson, Mitochondrial SIRT3 mediates adaptive responses of neurons to exercise and metabolic and excitatory challenges, Cell Metab. 23 (2016) 128-142. 
[69] M.L. Johnson, B.A. Irving, I.R. Lanza, M.H. Vendelbo, A.R. Konopka, M.M. Robinson, G.C. Henderson, K.A. Klaus, D.M. Morse, C. Heppelmann, H.R. Bergen 3rd, S. Dasari, J.M. Schimke, D.R. Jakaitis, K.S. Nair, Differential effect of endurance training on mitochondrial protein damage, degradation, and acetylation in the context of aging, J. Gerontol. A Biol. Sci. Med. Sci. 70 (2015) 1386-1393.

[70] E. Santos-Alves, I. Marques-Aleixo, P. Coxito, M.M. Balca, D. Rizo-Roca, S. RochaRodrigues, S. Martins, J.R. Torrella, P.J. Oliveira, A.J. Moreno, J. Magalhaes, A. Ascensao, Exercise mitigates diclofenac-induced liver mitochondrial dysfunction, Eur. J. Clin. Investig. 44 (2014) 668-677.

[71] H.K. Jiang, Y. Miao, Y.H. Wang, M. Zhao, Z.H. Feng, X.J. Yu, J.K. Liu, W.J. Zang, Aerobic interval training protects against myocardial infarction-induced oxidative injury by enhancing antioxidase system and mitochondrial biosynthesis, Clin. Exp. Pharmacol. Physiol. 41 (2014) 192-201.

[72] S.N. Blair, H.W. Kohl 3rd, R.S. Paffenbarger Jr, D.G. Clark, K.H. Cooper, L.W. Gibbons, Physical fitness and all-cause mortality. A prospective study of healthy men and women, JAMA 262 (1989) 2395-2401.

[73] X. Sui, M.J. LaMonte, J.N. Laditka, J.W. Hardin, N. Chase, S.P. Hooker, S.N. Blair, Cardiorespiratory fitness and adiposity as mortality predictors in older adults, JAMA 298 (2007) 2507-2516.

[74] Z. Radak, A.W. Taylor, H. Ohno, S. Goto, Adaptation to exercise-induced oxidative stress: from muscle to brain, Exerc. Immunol. Rev. 7 (2001) 90-107.

[75] T. Wyss-Coray, Ageing, neurodegeneration and brain rejuvenation, Nature 539 (2016) 180-186.

[76] G.A. Erikson, D.L. Bodian, M. Rueda, B. Molparia, E.R. Scott, A.A. Scott-Van Zeeland, S.E. Topol, N.E. Wineinger, J.E. Niederhuber, E.J. Topol, A. Torkamani, Whole-genome sequencing of a healthy aging cohort, Cell 165 (2016) 1002-1011.

[77] M.B. Schultz, D.A. Sinclair, When stem cells grow old: phenotypes and mechanisms of stem cell aging, Development 143 (2016) 3-14.

[78] J.M. Dickinson, P.T. Reidy, D.M. Gundermann, M.S. Borack, D.K. Walker, A.C. D'Lugos, E. Volpi, B.B. Rasmussen, The impact of postexercise essential amino acid ingestion on the ubiquitin proteasome and autophagosomal-lysosomal systems in skeletal muscle of older men, J. Appl. Physiol. 122 (2017) 620-630.

[79] Z. Radak, T. Kaneko, S. Tahara, H. Nakamoto, H. Ohno, M. Sasvari, C. Nyakas, S. Goto, The effect of exercise training on oxidative damage of lipids, proteins, and DNA in rat skeletal muscle: evidence for beneficial outcomes, Free Radic. Biol. Med. 27 (1999) 69-74.

[80] Z. Radak, T. Kaneko, S. Tahara, H. Nakamoto, J. Pucsok, M. Sasvari, C. Nyakas, S. Goto, Regular exercise improves cognitive function and decreases oxidative damage in rat brain, Neurochem. Int. 38 (2001) 17-23.

[81] V. Bonet-Costa, L.C. Pomatto, K.J. Davies, The proteasome and oxidative stress in Alzheimer's disease, Antioxid. Redox Signal. 25 (2016) 886-901.

[82] Z. Radak, N. Hart, L. Sarga, E. Koltai, M. Atalay, H. Ohno, I. Boldogh, Exercise plays a preventive role against Alzheimer's disease, J. Alzheimers Dis. 20 (2010) 777-783.

[83] I.J. Vechetti-Junior, R.S. Bertaglia, G.J. Fernandez, T.G. de Paula, R.W. de Souza, L.N. Moraes, E.A. Mareco, C.E. de Freitas, A.F. Aguiar, R.F. Carvalho, M. Dal-PaiSilva, Aerobic exercise recovers disuse-induced atrophy through the stimulus of the LRP130/PGC-1alpha complex in aged rats, J. Gerontol. A Biol. Sci. Med. Sci. 71 (2016) 601-609.

[84] M.M. Ziaaldini, E. Koltai, Z. Csende, S. Goto, I. Boldogh, A.W. Taylor, Z. Radak, Exercise training increases anabolic and attenuates catabolic and apoptotic processes in aged skeletal muscle of male rats, Exp. Gerontol. 67 (2015) 9-14.

[85] U. Raue, D. Slivka, B. Jemiolo, C. Hollon, S. Trappe, Proteolytic gene expression differs at rest and after resistance exercise between young and old women, J. Gerontol. A Biol. Sci. Med. Sci. 62 (2007) 1407-1412.

[86] Z. Sha, A.L. Goldberg, Proteasome-mediated processing of Nrf1 is essential for coordinate induction of all proteasome subunits and p97, Curr. Biol. 24 (2014) 1573-1583.

[87] K.L. Rock, C. Gramm, L. Rothstein, K. Clark, R. Stein, L. Dick, D. Hwang, A.L. Goldberg, Inhibitors of the proteasome block the degradation of most cell proteins and the generation of peptides presented on MHC class I molecules, Cell 78 (1994) 761-771.

[88] B.L. Lee, A. Singh, J.N. Mark Glover, M.J. Hendzel, L. Spyracopoulos, Molecular basis for k63-linked ubiquitination processes in double-strand DNA break repair: a focus on kinetics and dynamics, J. Mol. Biol. 429 (2017) 3409-3429.

[89] D. Shi, W. Gu, Dual roles of MDM2 in the regulation ofp53: ubiquitination dependent and ubiquitination independent mechanisms of MDM2 repression of p53 activity, Genes Cancer 3 (2012) 240-248.

[90] B. Ferrando, M.C. Gomez-Cabrera, A. Salvador-Pascual, C. Puchades, F. Derbre, A. Gratas-Delamarche, L. Laparre, G. Olaso-Gonzalez, M. Cerda, E. Viosca, A. Alabajos, V. Sebastia, A. Alberich-Bayarri, F. Garcia-Castro, J. Vina, Allopurinol partially prevents disuse muscle atrophy in mice and humans, Sci. Rep. 8 (2018) 3549.

[91] D.A. Bota, K.J. Davies, Lon protease preferentially degrades oxidized mitochondrial aconitase by an ATP-stimulated mechanism, Nat. Cell Biol. 4 (2002) 674-680.

[92] D.A. Bota, H. Van Remmen, K.J. Davies, Modulation of Lon protease activity and aconitase turnover during aging and oxidative stress, FEBS Lett. 532 (2002) 103-106.

[93] A. Wagatsuma, N. Kotake, T. Kawachi, M. Shiozuka, S. Yamada, R. Matsuda, Mitochondrial adaptations in skeletal muscle to hindlimb unloading, Mol. Cel Biochem. 350 (2011) 1-11.

[94] O. Marton, E. Koltai, M. Takeda, L.G. Koch, S.L. Britton, K.J. Davies, I. Boldogh, Z. Radak, Mitochondrial biogenesis-associated factors underlie the magnitude of response to aerobic endurance training in rats, Pflug. Arch. 467 (2015) 779-788.
[95] S. Nicolai, A. Rossi, N. Di Daniele, G. Melino, M. Annicchiarico-Petruzzelli, G. Raschella, DNA repair and aging: the impact of the p53 family, Aging 7 (2015) 1050-1065.

[96] A. Safdar, J.M. Bourgeois, D.I. Ogborn, J.P. Little, B.P. Hettinga, M. Akhtar, J.E. Thompson, S. Melov, N.J. Mocellin, G.C. Kujoth, T.A. Prolla,

M.A. Tarnopolsky, Endurance exercise rescues progeroid aging and induces systemic mitochondrial rejuvenation in mtDNA mutator mice, Proc. Natl. Acad. Sci. USA 108 (2011) 4135-4140.

[97] A. Safdar, K. Khrapko, J.M. Flynn, A. Saleem, M. De Lisio, A.P. Johnston, Y. Kratysberg, I.A. Samjoo, Y. Kitaoka, D.I. Ogborn, J.P. Little, S. Raha, G. Parise, M. Akhtar, B.P. Hettinga, G.C. Rowe, Z. Arany, T.A. Prolla, M.A. Tarnopolsky, Exercise-induced mitochondrial p53 repairs mtDNA mutations in mutator mice, Skelet. Muscle 6 (2016) 7.

[98] J.D. Bartlett, G.L. Close, B. Drust, J.P. Morton, The emerging role of p53 in exercise metabolism, Sports Med. 44 (2014) 303-309.

[99] A.W. Lau, P. Liu, H. Inuzuka, D. Gao, SIRT1 phosphorylation by amp-activated protein kinase regulates p53 acetylation, Am. J. Cancer Res. 4 (2014) 245-255.

[100] J.N. Feige, M. Lagouge, C. Canto, A. Strehle, S.M. Houten, J.C. Milne, P.D. Lambert, C. Mataki, P.J. Elliott, J. Auwerx, Specific SIRT1 activation mimics low energy levels and protects against diet-induced metabolic disorders by enhancing fat oxidation, Cell Metab. 8 (2008) 347-358.

[101] D.C. Rubinsztein, G. Marino, G. Kroemer, Autophagy and aging, Cell 146 (2011) 682-695.

[102] A. Vainshtein, L.D. Tryon, M. Pauly, D.A. Hood, Role of PGC-1alpha during acute exercise-induced autophagy and mitophagy in skeletal muscle, Am. J. Physiol. Cell Physiol. 308 (2015) C710-C719.

[103] A.J. Smuder, K.J. Sollanek, W.B. Nelson, K. Min, E.E. Talbert, A.N. Kavazis, M.B. Hudson, M. Sandri, H.H. Szeto, S.K. Powers, Crosstalk between autophagy and oxidative stress regulates proteolysis in the diaphragm during mechanical ventilation, Free Radic. Biol. Med. 115 (2018) 179-190.

[104] A. Vainshtein, D.A. Hood, The regulation of autophagy during exercise in skeletal muscle, J. Appl. Physiol. 120 (2016) 664-673.

[105] Y. Kiriyama, H. Nochi, Intra- and intercellular quality control mechanisms of mitochondria, Cells 7 (2017).

[106] D.F. Egan, D.B. Shackelford, M.M. Mihaylova, S. Gelino, R.A. Kohnz, W. Mair, D.S. Vasquez, A. Joshi, D.M. Gwinn, R. Taylor, J.M. Asara, J. Fitzpatrick, A. Dillin, B. Viollet, M. Kundu, M. Hansen, R.J. Shaw, Phosphorylation of ULK1 (hATG1) by AMP-activated protein kinase connects energy sensing to mitophagy, Science 331 (2011) 456-461.

[107] W.J. Smiles, M.S. Conceicao, G.D. Telles, M.P. Chacon-Mikahil, C.R. Cavaglieri, F.C. Vechin, C.A. Libardi, J.A. Hawley, D.M. Camera, Acute low-intensity cycling with blood-flow restriction has no effect on metabolic signaling in human skeletal muscle compared to traditional exercise, Eur. J. Appl. Physiol. 117 (2017) 345-358.

[108] C. Jamart, D. Naslain, H. Gilson, M. Francaux, Higher activation of autophagy in skeletal muscle of mice during endurance exercise in the fasted state, Am. J. Physiol. Endocrinol. Metab. 305 (2013) E964-E974.

[109] R.C. Laker, J.C. Drake, R.J. Wilson, V.A. Lira, B.M. Lewellen, K.A. Ryall, C.C. Fisher, M. Zhang, J.J. Saucerman, L.J. Goodyear, M. Kundu, Z. Yan, Ampk phosphorylation of Ulk1 is required for targeting of mitochondria to lysosomes in exercise-induced mitophagy, Nat. Commun. 8 (2017) 548.

[110] R.J. Thomas, S.A. Kenfield, A. Jimenez, Exercise-induced biochemical changes and their potential influence on cancer: a scientific review, Br. J. Sports Med. 51 (2017) 640-644.

[111] M. Dizdaroglu, Formation of an 8-hydroxyguanine moiety in deoxyribonucleic acid on gamma-irradiation in aqueous solution, Biochemistry 24 (1985) 4476-4481.

[112] I. Boldogh, G. Hajas, L. Aguilera-Aguirre, M.L. Hegde, Z. Radak, A. Bacsi, S. Sur, T.K. Hazra, S. Mitra, Activation of ras signaling pathway by 8-oxoguanine DNA glycosylase bound to its excision product, 8-oxoguanine, J. Biol. Chem. 287 (2012) 20769-20773.

[113] H. Sampath, V. Vartanian, M.R. Rollins, K. Sakumi, Y. Nakabeppu, R.S. Lloyd, 8 Oxoguanine DNA glycosylase (OGG1) deficiency increases susceptibility to obesity and metabolic dysfunction, PLoS One 7 (2012) e51697.

[114] L. Pan, B. Zhu, W. Hao, X. Zeng, S.A. Vlahopoulos, T.K. Hazra, M.L. Hegde, Z. Radak, A. Bacsi, A.R. Brasier, X. Ba, I. Boldogh, Oxidized guanine base lesions function in 8-oxoguanine DNA Glycosylase-1-mediated epigenetic regulation of nuclear factor kappaB-driven gene expression, J. Biol. Chem. 291 (2016) 25553-25566.

[115] M. Seifermann, B. Epe, Oxidatively generated base modifications in DNA: not only carcinogenic risk factor but also regulatory mark? Free Radic. Biol. Med. 107 (2017) 258-265.

[116] A.M. Fleming, C.J. Burrows, 8-Oxo-7,8-dihydroguanine, friend and foe: epigenetic-like regulator versus initiator of mutagenesis, DNA Repair 56 (2017) 75-83.

[117] M. Daimon, T. Oizumi, S. Toriyama, S. Karasawa, Y. Jimbu, K. Wada, W. Kameda S. Susa, M. Muramatsu, I. Kubota, S. Kawata, T. Kato, Association of the Ser326Cys polymorphism in the OGG1 gene with type 2 DM, Biochem. Biophys. Res. Commun. 386 (2009) 26-29.

[118] Z. Radak, H. Naito, T. Kaneko, S. Tahara, H. Nakamoto, R. Takahashi, F. CardozoPelaez, S. Goto, Exercise training decreases DNA damage and increases DNA repair and resistance against oxidative stress of proteins in aged rat skeletal muscle, Pflug. Arch. 445 (2002) 273-278

[119] H. Nakamoto, T. Kaneko, S. Tahara, E. Hayashi, H. Naito, Z. Radak, S. Goto, Regular exercise reduces 8-oxodG in the nuclear and mitochondrial DNA and modulates the DNA repair activity in the liver of old rats, Exp. Gerontol. 42 (2007) 287-295 
[120] C. Tweedie, C. Romestaing, Y. Burelle, A. Safdar, M.A. Tarnopolsky, S. Seadon, S.L. Britton, L.G. Koch, R.T. Hepple, Lower oxidative DNA damage despite greater ROS production in muscles from rats selectively bred for high running capacity, Am. J. Physiol. Regul. Integr. Comp. Physiol. 300 (2011) R544-R553.

[121] Z. Radak, P. Apor, J. Pucsok, I. Berkes, H. Ogonovszky, G. Pavlik, H. Nakamoto, S. Goto, Marathon running alters the DNA base excision repair in human skeletal muscle, Life Sci. 72 (2003) 1627-1633.

[122] K.K. Bhakat, S.K. Mokkapati, I. Boldogh, T.K. Hazra, S. Mitra, Acetylation of human 8-oxoguanine-DNA glycosylase by p300 and its role in 8-oxoguanine repair in vivo, Mol. Cell. Biol., 26, 2006, pp.1654-1665.

[123] L. Sarga, N. Hart, L.G. Koch, S.L. Britton, G. Hajas, I. Boldogh, X. Ba, Z. Radak, Aerobic endurance capacity affects spatial memory and SIRT1 is a potent modulator of 8-oxoguanine repair, Neuroscience 252 (2013) 326-336.
[124] M. Torres-Gonzalez, T. Gawlowski, H. Kocalis, B.T. Scott, W.H. Dillmann, Mitochondrial 8-oxoguanine glycosylase decreases mitochondrial fragmentation and improves mitochondrial function in $\mathrm{H} 9 \mathrm{C} 2$ cells under oxidative stress conditions, Am. J. Physiol. Cell Physiol. 306 (2014) C221-C229.

[125] V. Vartanian, J. Tumova, P. Dobrzyn, A. Dobrzyn, Y. Nakabeppu, R.S. Lloyd, H. Sampath, 8-oxoguanine DNA glycosylase (OGG1) deficiency elicits coordinated changes in lipid and mitochondrial metabolism in muscle, PLoS One 12 (2017) e0181687.

[126] Z. Radak, M. Atalay, J. Jakus, I. Boldogh, K. Davies, S. Goto, Exercise improves import of 8-oxoguanine DNA glycosylase into the mitochondrial matrix of skeletal muscle and enhances the relative activity, Free Radic. Biol. Med. 46 (2009) 238-243. 